\title{
Echocardiographic Imaging and Quantification of Myocardial Perfusion Based on Interrupted Contrast Infusion
}

\author{
E Toledo, RM Lang, KA Collins, U Williams, \\ G Lammertin, G Bolotin, V Mor-Avi \\ University of Chicago, Chicago, IL, USA
}

\begin{abstract}
Echocardiographic quantification of myocardial perfusion, based on analysis of contrast replenishment following destructive high-energy ultrasound impulses (flash-echo), has multiple limitations. We present an alternative approach, based on analysis of contrast replenishment after brief interruptions of contrast infusion (ICI). Images were acquired in 8 isolated rabbit hearts at 3 flow levels (baseline, 50\% and 15\%) during contrast infusion (Definity) with flash-echo and, after ICI. Peak contrast inflow rate (PCIR) was calculated from ICI data and compared with flash-echo data. Clinical feasibility was tested in 5 subjects with the ICI technique at rest and stress. In rabbit hearts, PCIR followed changes in coronary flow $(p<0.0001)$ and had lower inter-measurement variability than the flash-echo data. In humans, stress PCIR was $284 \pm 142 \%$ of resting value. ICI provides the basis for accurate and reproducible quantification of myocardial perfusion and may constitute an alternative to the currently used techniques.
\end{abstract}

\section{Introduction}

Quantitative assessment of myocardial perfusion from echocardiographic images has several advantages over other existing techniques, including wide availability, portability, low cost and no radiation concerns. Today, echocardiographic assessment of myocardial perfusion is based on analysis of contrast replenishment following high-energy impulses, which are delivered to destroy microbubbles in the myocardium (flash-echo) [1]. According to indicator dilution principles, post-flash contrast replenishment has an exponential form:

$$
m v i(t) \approx A\left(1-e^{-\beta_{0}\left(t-t_{0}\right)}\right)+C
$$

where $\operatorname{MVI}(t)$ is the mean videointensity, $t_{0}$ is the onset of contrast replenishment, $C$ is the pre-contrast videointensity level, $A$ is the contrast-induced enhancement and $\beta=$ (blood flow) $/$ (volume of distribution). Quantification of perfusion is achieved by fitting such a model to MVI curves. The problems of flash-echo are: (i) uniform bubble destruction is often difficult to achieve; (ii) microbubble destruction occurs only in a thin slice representing the imaging plane, resulting in fast contrast replenishment (typically 3-4 cardiac cycles), and thus severely limiting the accuracy of this technique.

We hypothesized that a brief interruption in contrast infusion (ICI) would result in uniform contrast clearance from the entire myocardium followed by gradual replenishment once the infusion is resumed. As a result, the contrast replenishment phase would be longer and thus the calculation of inflow rates would be more accurate and less affected by noise. This study was designed to initially test this hypothesis in an isolated heart model that allows full control of coronary flow and provides near ideal imaging conditions. Subsequently, we tested the clinical feasibility of this approach in humans.

\section{Methods}

\subsection{Isolated heart experiments}

Animal preparation. Experiments were performed in 8 rabbits $(2.2-2.6 \mathrm{~kg})$. Under anesthesia, the heart was removed from the body, attached to a Langendorff apparatus, and perfused retrogradely via the aortic root with Krebs-Henseleit solution at constant perfusion pressure $(86 \mathrm{mmHg})$. Global coronary flow was continuously monitored. A thin latex balloon was introduced into the left ventricular (LV) cavity via the mitral annulus and filled with fluid to allow the heart generate physiologic pressures during isovolumic contractions. The heart was immersed in warm solution in a plastic container to allow imaging through the wall.

Ultrasound imaging. ECG-triggered imaging (Philips 7500) was performed during contrast infusion of Definity (16-25 $\mathrm{ml} / \mathrm{hr}$, Bristol-Myers Squibb) into the perfusion line just proximal to the heart. The rate of infusion was set to maximize myocardial contrast without attenuation in the distal myocardium. ICI image sequences were obtained using an S12 transducer (5-12 MHz). An S3 transducer (2.5 MHz, power modulation) was used to acquire flash-echo sequences from the same transducer position. 
Protocol. Three consecutive ICI image sequences were acquired at baseline (BL) conditions over 1 min each, including $10 \mathrm{sec}$ steady state, 20 to $25 \mathrm{sec}$ interruption of infusion followed by $30 \mathrm{sec}$ of contrast inflow to return to a steady state. Image acquisition was then repeated at two levels of reduced coronary flow: $50 \%$ of BL flow (F1; 40$60 \%$ were acceptable), and $15 \%$ of BL (F2; $10-20 \%$ were acceptable). Subsequently, three consecutive flash-echo sequences were acquired at BL, including $10 \mathrm{sec}$ steady state, followed by a high-energy impulse, and $30 \mathrm{sec}$ of post-impulse contrast replenishment. Image acquisition was then repeated 3 times at each F1 and F2 flow.

Image analysis. A region of interest (ROI) that included the LV myocardium was defined manually. Image data in this ROI was analyzed differently for ICI and flash-echo sequences. Flash-echo sequences were analyzed using a previously described technique [1]. Briefly, MVI was calculated by averaging pixel intensity in the entire ROI for each consecutive frame to generate an MVI(t) curves. Exponential function was fitted to the replenishment portion of the curve, beginning at the lowest post-flash point. The calculated constant of the exponential function, $\beta$, an estimate of $\beta_{0}$, was used as a perfusion index. For each frame in the ICI sequences, local MVI was calculated for each pixel and filtered using a $5 \times 5$ circular Gaussian low-pass filter. The resultant local MVI(t) curve was used to estimate contrast-induced change for each pixel. Time-derivative of the local MVI $(\mathrm{t})$ was obtained from a $2^{\text {nd }}$ order Savitzky-Golay polynomial fit using a moving window of 11 points. The maximum of the timederivative divided by the local contrast-induced MVI change was calculated and averaged over the entire ROI, while excluding $20 \%$ outlier values. This step resulted in peak contrast inflow rate (PCIR), which was used as an estimate of $\beta_{0}$. In addition, signal-to-noise ratio (SNR) was calculated for both flash-echo and ICI sequences.

Statistical analysis. Changes in PCIR and $\beta$ from BL were tested using paired $\mathrm{t}$-test. The inter-measurement variability of each perfusion index was calculated for each animal at each level of coronary flow as the SD of
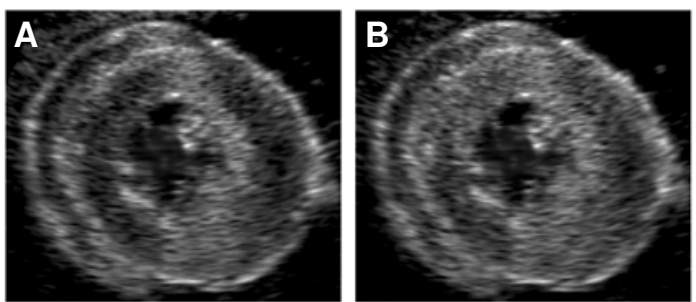

the repeated measurements and expressed in \% of their mean. The differences between inter-measurement variability of PCIR and $\beta$ were tested using paired t-test.

\subsection{Computer simulation}

Simulated curves were used to investigate the effects of noise on the accuracy of the calculations of PCIR and $\beta$. A time series was created to simulate MVI changes during contrast inflow based on eq. (1), where the constants were assigned values resulting in approximately 6-25 time points in the upslope phase, depending on the specific value of $\beta_{0}$. For each time series, random Gaussian white noise was added at levels corresponding to SNR ranging from 5 to 50 . Each simulated time series was analyzed using: (i) exponential fitting; (ii) PCIR calculation from the time derivative. For each combination of $\beta_{\mathrm{o}}$ and SNR, this procedure was repeated 5000 times and the SD of the values was expressed in \% of their mean to reflect the variability of each algorithm at each noise level. Since the identification of the starting point $t_{0}$, necessary for exponential fitting, can be inaccurate in the presence of noise, we studied the effects of erroneously detecting $t_{\mathrm{o}}$ on the calculated values of $\beta$. The simulated time series with the different levels of noise were reanalyzed while shifting $t_{\mathrm{o}} 1-4$ points in each direction. This procedure was repeated 100 times for each combination of $\beta_{\mathrm{o}}$, SNR and time shift. The bias between the mean $\beta$ and the true $\beta_{\mathrm{o}}$ was expressed in percent of $\beta_{\mathrm{o}}$.

\subsection{Human studies}

Imaging was performed in 8 normal subjects $(6 \mathrm{~F}$, age $45 \pm 8)$. ICI sequences were obtained and analyzed at rest, and during adenosine stress $(0.142 \mathrm{ml} / \mathrm{Kg} / \mathrm{min})$. To compensate for recirculation and differences in rates of transpulmonary contrast passage, myocardial PCIR was normalized by PCIR calculated in the LV cavity.

\section{Results}

In isolated rabbit hearts, global coronary flow was $45 \pm 9 \mathrm{ml} / \mathrm{min}$ at BL. Imaging of the isolated heart resulted in high quality images with the spatial resolution varying

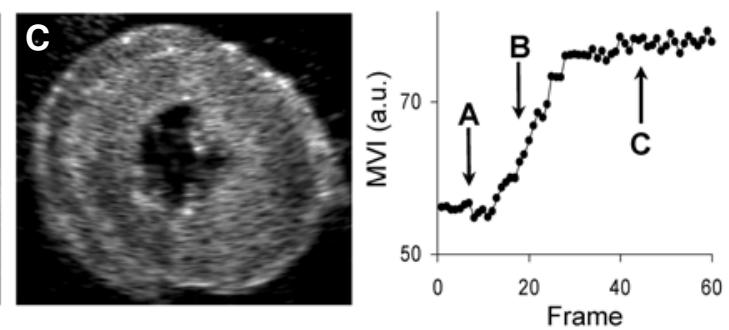

Figure 1. Example of images of the isolated rabbit heart obtained using a wide-band (5-12 MHz) transducer during an interrupted contrast infusion (ICI) sequence at baseline coronary flow: (A) at peak contrast clearance, (B) during contrast wash-in, and (C) after reestablishing steady-state contrast. Labels A-C on the myocardial videointensity time curve (right) depict the time points in the sequence at which the 3 images were obtained. 

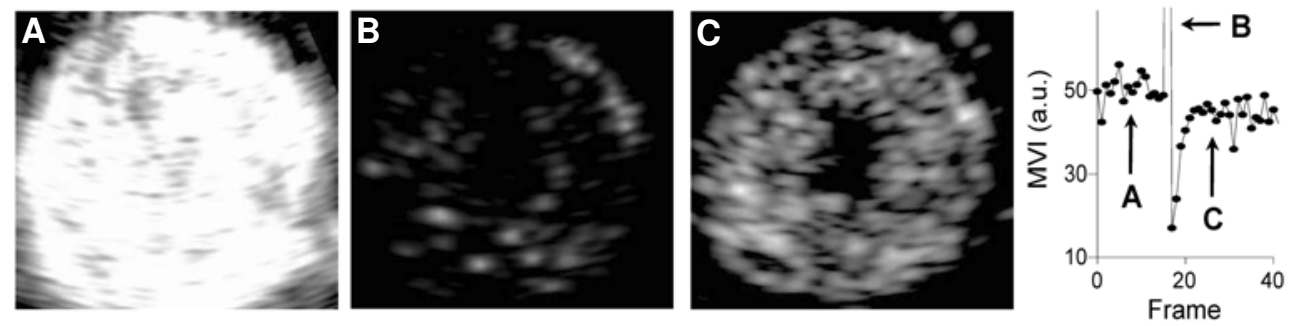

Figure 2. Example of images obtained using a $2.5 \mathrm{MHz}$ transducer at baseline coronary flow: (A) during high-energy impulse, (B) during peak bubble destruction, immediately after the impulse and (C) after contrast replenishment.

between the transducers. ICI resulted in visible and uniform contrast clearance (fig 1). Contrast inflow occurred within 15-25 cardiac cycles at BL, and was longer at lower coronary flows. SNR levels varied from 6 to 40 with the highest values measured at BL. With flashecho, uniform bubble destruction and replenishment were clearly visible (fig 2), despite the low spatial resolution. Post-impulse contrast replenishment occurred within of 4 to 6 cardiac cycles at $\mathrm{BL}$ and was longer at lower coronary flows. SNR levels varied from 4 to 38 without clear dependence on flow. Both $\beta$ and PCIR followed the changes in coronary flow (fig 3 ). While these changes were significant for $\beta$ only at F2, changes in PCIR were significant even at F1. The inter-animal variability reflected by the ratio between the size of the error bars and the mean values (fig 3$)$ was higher for $\beta(\approx 100 \%)$ than for PCIR $(\approx 20 \%)$. Inter-measurement variability was $42 \pm 33 \%$ for $\beta$ and $7 \pm 6 \%$ for PCIR ( $<<0.001)$.

Analysis of simulated data showed that the computational errors of both $\beta$ and PCIR were directly related to noise level (fig 4). In the presence of noise, the variability in $\beta$ was twice as high as that of PCIR. Incorrectly identifying $t_{0}$ biased the values of $\beta$. The magnitude of the bias was related to both the time shift and the noise level (fig 5), reaching over $100 \%$ of the true $\beta_{0}$ for the noisier curves, when $t_{0}$ was shifted $>2$ points.

In all humans, dynamic changes in MVI were visible and quantifiable. Visible myocardial contrast clearance occurred within 90sec. The transition from no enhancement to steady-state enhancement occurred in all subjects within $<45 \mathrm{sec}$, and was captured in a single data acquisition. Adenosine resulted an increase in the rate of contrast inflow in all subjects. Consequently, PCIR with adenosine was $284 \pm 142 \%$ of the resting value.

\section{Discussion and conclusions}

The ability to quantify myocardial perfusion relies on the notion of dynamic changes in tissue contrast. Initially, short intravenous boluses of contrast media were used to estimate perfusion $[2,3]$. This approach was plagued by the need to guess a priori the optimal imaging settings for visualizing the short-lived one-time event of the bolus
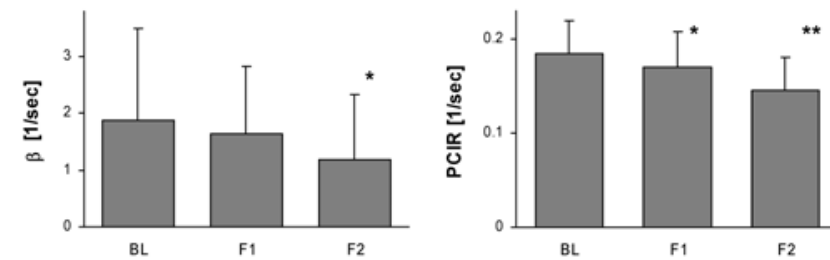

Figure 3. Calculated values of myocardial perfusion indices: $\beta$ obtained from flash-echo sequences (left) and PCIR obtained from ICI sequences (right) at different levels of coronary flow (BL: baseline, F1: $50 \%$ of BL flow, F2: $15 \%$ BL flow), averaged over all animals. $\left({ }^{*} \mathrm{p}<0.05\right.$ and ${ }^{* *} \mathrm{p}<0.001$ versus BL). See text for details.
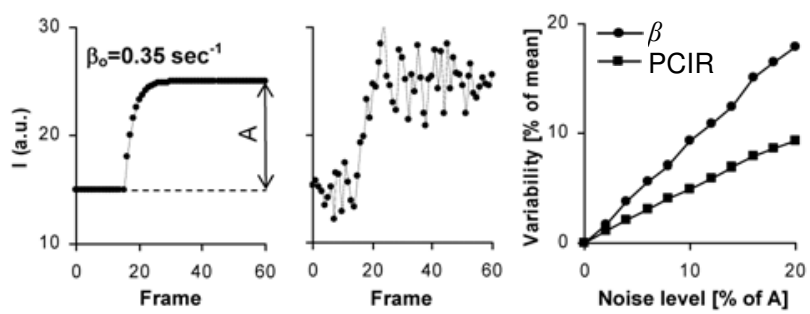

Figure 4. Simulated contrast inflow curves with different SNR levels (left and middle). Variability in calculated values of $\beta$ and PCIR as a function of noise (right).

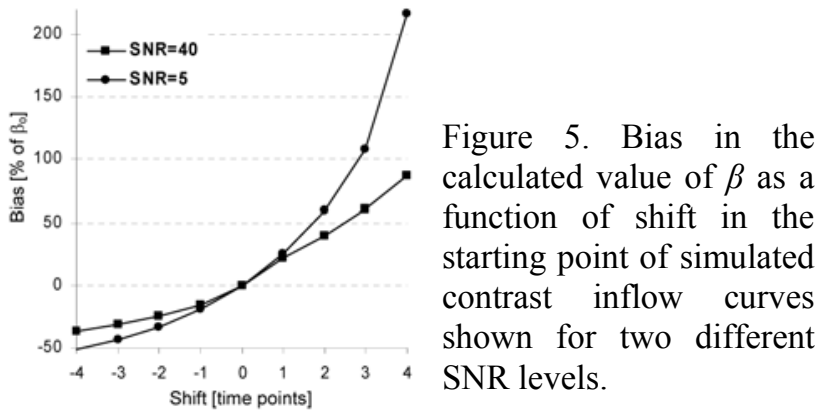

traversing the heart. To overcome this difficulty, new contrast agents were developed for continuous infusion resulting in steady-state contrast enhancement. To achieve dynamic changes in tissue contrast, flash-echo utilized the destruction of microbubbles, the subsequent replenishment of which is related to tissue blood flow 
$[1,4]$. Since in our experience, the accuracy and reproducibility of flash-echo are limited, we sought to develop alternative approaches to both acquisition and analysis. This study was designed to initially test such an alternative in an isolated heart, optimize its performance on simulated data, and then test its clinical feasibility.

Brief interruption of contrast infusion as acquisition strategy has several advantages over both contrast boluses and infusion with flash-echo. While ICI provides virtually unlimited time for optimization of gain settings unlike contrast boluses, it also induces the dynamic changes in myocardial contrast, without the need for high-energy pulses with their limitations. Since ICI clears the entire myocardium, the slow rate of subsequent contrast inflow offers the advantage of a higher number of time points. When combined with noise that is impossible to avoid, such increased number of data points warrants improved accuracy and reproducibility, as proved by our results.

Our analysis procedure included two elements that were substantially different from previous techniques. Rather than calculating mean pixel intensity in a myocardial ROI over time, we created a time series of local videointensity, from which flow information was extracted and averaged for the entire ROI. This allowed us to discard information originating from outlier pixels and thus reduce artifacts. Second, exponential fitting is sensitive to noise and to inaccurate identification of the starting point. Accordingly, we sought for a quantitative perfusion index that could be accurately and reproducibly obtained from contrast inflow curves without exponential fitting. Since the maximum of the MVI(t) time-derivative equals $A \cdot \beta_{0}$, we used it to extract $\beta_{\mathrm{o}}$. The relatively large number of points allowed the use of time-localized polynomial approximation for numerical computation of the time-derivative. Since this procedure did not require the identification of $t_{0}$, this source of error was eliminated. The results of our simulation proved the advantages of this approach over exponential fitting.

Our approach, based on analysis of ICI sequences was found sufficiently sensitive to detect a $50 \%$ reduction in coronary flow, which would not even be considered clinically significant. This technique was also found to have low inter-animal variability and high reproducibility. Since the image analysis procedure is fully automated, inter- and intra-observer variability were not an issue.

A limitation of our study is the different imaging frequencies of the transducers, which resulted in superior spatial resolution of the ICI. Our choice of the highfrequency transducer for the small rabbit heart was a necessity for optimizing the conditions for developing this new concept. On the other hand, flash-echo required a capability of transmitting high-energy pulses, only available with the low frequency transducers. We found that the SNR in MVI curves was not different with the two probes, probably due to the use of power modulation with the low frequency probe. Therefore, it is likely that this choice of equipment did not bias our results.

The human protocol was aimed at initial testing of the applicability of the ICI technique in a clinical setting. This was achieved by measuring pharmacologically induced changes in myocardial perfusion. This strategy was chosen because the ultimate target clinical application of this approach is the detection of stressinduced perfusion abnormalities. The use of normal subjects allowed us to validate our technique against the anticipated normal response to adenosine, i.e. a marked increase in perfusion in the absence of coronary artery disease. Our results demonstrated a three-fold increase in the measured perfusion index caused by adenosine. We also found that contrast clearance and inflow occur within a timeframe applicable in the clinical setting.

The ability of PCIR obtained from ICI sequences to track changes in myocardial flow demonstrated the sensitivity and accuracy of this technique. Additionally, our results showed that PCIR is a highly reproducible index of myocardial perfusion with low inter-animal variability. This technique may thus constitute an alternative to the currently used flash-echo techniques. The results of our human studies indicate that the ICI technique can be used in humans, and establish the basis for future clinical studies geared toward the validation of this technique in different cardiac disease states.

\section{References}

[1] Wei K, Jayaweera AR, Firoozan S, Linka A, Skyba DM, Kaul S. Quantification of myocardial blood flow with ultrasound-induced destruction of microbubbles administered as a constant venous infusion. Circulation. 1998;97:473-83.

[2] Mor-Avi V, David D, Akselrod S, Bitton Y, Choshniak I. Myocardial regional blood flow: quantitative measurement by computer analysis of contrast enhanced echocardiographic images. Ultrasound Med Biol. 1993;19:619-33.

[3] Skyba DM, Jayaweera AR, Goodman NC, Ismail S, Camarano G, Kaul S. Quantification of myocardial perfusion with myocardial contrast echocardiography during left atrial injection of contrast. Implications for venous injection. Circulation. 1994;90:1513-21.

[4] Masugata H, Peters B, Lafitte S, Strachan GM, Ohmori K, DeMaria AN. Quantitative assessment of myocardial perfusion during graded coronary stenosis by real-time myocardial contrast echo refilling curves. J Am Coll Cardiol. 2001;37:262-69.

Address for correspondence

Victor Mor-Avi

University of Chicago Medical Center, MC5084, 5841 S. Maryland Ave., Chicago Illinois 60637, USA

vmoravi@medicine.bsd.uchicago.edu 